\title{
Simulation of Gas-Phase Chemistry for Selected Carbon Precursors in Epitaxial Growth of SiC
}

\author{
Örjan Danielsson, Pitsiri Sukkaew, Milan Yazdanfar, Olle Kordina and Erik Janzén
}

\section{Linköping University Post Print}

\section{Tweet}

N.B.: When citing this work, cite the original article.

Original Publication:

Örjan Danielsson, Pitsiri Sukkaew, Milan Yazdanfar, Olle Kordina and Erik Janzén, Simulation of Gas-Phase Chemistry for Selected Carbon Precursors in Epitaxial Growth of SiC, 2013, Materials Science Forum, (740-742), 213-216.

http://dx.doi.org/10.4028/www.scientific.net/MSF.740-742.213

Copyright: Trans Tech Publications

http://www.ttp.net/

Postprint available at: Linköping University Electronic Press

http://urn.kb.se/resolve?urn=urn:nbn:se:liu:diva-96506 


\title{
Simulation of gas-phase chemistry for selected carbon precursors in epitaxial growth of SiC
}

\author{
Örjan Danielsson ${ }^{1, a}$, Pitsiri Sukkaew ${ }^{1, b}$, Milan Yazdanfar ${ }^{1, c}$, \\ Olof Kordina ${ }^{1, d}$ and Erik Janzén ${ }^{1, e}$
}

\author{
${ }^{1}$ Department of Physics, Chemistry and Biology, \\ Linköping University, SE - 58183 Linköping, Sweden \\ aorjda@ifm.liu.se, bpitsu@ifm.liu.se, milya@ifm.liu.se, olkor@ifm.liu.se, erj@ifm.liu.se
}

\begin{abstract}
Keywords: Epitaxial growth, Chemical Vapor Deposition, Modeling, Simulation, Gas-phase chemistry, Hydrocarbons
\end{abstract}

\begin{abstract}
Numerical simulations are one way to obtain a better and more detailed understanding of the chemical vapor deposition process of silicon carbide. Although several attempts have been made in this area during the past ten years, there is still no general model valid for any range of process parameters and choice of precursors, that can be used to control the growth process, and to optimize growth equipment design. In this paper a first step towards such a model is taken. Here, mainly the hydrocarbon chemistry is studied by a detailed gas-phase reaction model, and comparison is made between $\mathrm{C}_{3} \mathrm{H}_{8}$ and $\mathrm{CH}_{4}$ as carbon precursor. The results indicate that experimental differences, which previous models have been unable to predict, may be explained by the new model.
\end{abstract}

\section{Introduction}

Understanding the chemistry of chemical vapor deposition (CVD) of SiC is important to be able to improve and control the growth process, and to optimize growth equipment design. Using numerical simulations, e.g. computational fluid dynamics (CFD), is one way of obtaining this understanding. During the past ten years such methods have successfully been used to simulate both gas-phase and surface chemistry for epitaxial growth of SiC by CVD [1 - 4]. However, the models have often been validated for one particular set of process parameters in one particular growth equipment. Therefore, it is uncertain if these models give accurate predictions outside the conditions used.

To be really useful, a model of the epitaxial growth of $\mathrm{SiC}$ should take into account all important species and all important reaction paths, and be valid for all parameter ranges of interest. One evident proof of the shortcomings of the existing models for CVD growth of SiC is the inability to correctly model the hydrocarbon chemistry. This is somewhat surprising, since the hydrocarbon chemistry is thoroughly investigated for combustion processes, see e.g. [5, 6].

For the most commonly used process parameters and precursors the epitaxial growth of $\mathrm{SiC}$ takes place in a silicon limited regime. Thus, very accurate results can be obtained by only including reactions between silicon containing species in the chemical models [1]. It has also been found that the two most common precursor combinations, $\mathrm{SiH}_{4}+\mathrm{C}_{3} \mathrm{H}_{8}$ and $\mathrm{SiH}_{4}+\mathrm{C}_{2} \mathrm{H}_{4}$, give similar concentration profiles along the gas flow direction for simplified models [7]. Therefore very little effort has been made to investigate the reaction paths for hydrocarbons further.

Experimentally it is found that there is a large difference in material quality depending on the carbon precursor used [8], even though growth rates are similar. And when using chlorinated precursors, precursor flow rates need to be altered to obtain high quality layers. This sometimes leads to a carbon limited growth regime [9]. Better and more accurate models can help to explain these differences and understand the chemistry in SiC epitaxial growth by CVD in more detail. 


\section{Model setup}

As a starting point for compiling a more general, and hopefully more useful, model for the epitaxial growth of $\mathrm{SiC}$, a detailed gas-phase reaction model for the hydrocarbon chemistry is set up here, including 24 species $\left(\mathrm{C}_{\mathrm{x}} \mathrm{H}_{\mathrm{y}}\right.$ up to $\mathrm{x}=3$, plus $\mathrm{H}$ and $\left.\mathrm{H}_{2}\right)$, consisting of more than 180 different elementary reaction steps. Reaction rates are mainly taken from Tsang et al. [5, 6], but also from several more recent works. In addition the model of Ho et al. [10] is included to account for reactions between silicon species, with the purpose to be able to determine if the deposition is limited by carbon or silicon. The gas-phase model of Ho for the silicon species was chosen because it has been shown previously that it can give very accurate results for deposition rates in a silicon limited growth regime [1]. At this stage no organosilicon species (i.e. species containing both carbon and silicon) or reactions between silanes and hydrocarbons are included in the model.

A simple surface reaction model is included to take into account the depletion effect along the gas flow direction, and the resulting deposition rates are used as a way to compare the different models and precursors. This simple surface reaction model is based on sticking probabilities, which have been used with some success in previous works to predict growth rates in silicon limited growth regimes $[1,2]$. It should be noted, however, that the choice of surface reaction model will have a significant impact on the resulting deposition rates, and therefore only relative values will be presented here.

It is assumed in the model that silicon and carbon are deposited independently of each other; an approach used in several previous work on modeling of SiC CVD growth [1, 11]. In the calculations it is also assumed that the density of the deposited layer is constant over the whole deposition area.

The chemical reaction models are implemented in commercial CFD software and tested in a 2D geometry of a horizontally oriented CVD reactor. A temperature profile based on measured values was set on the boundaries of the model, with a maximum temperature of $1600^{\circ} \mathrm{C}$ at the wafer position. $\mathrm{SiH}_{4}$ is used as silicon precursor, while two different carbon precursors are compared. An inlet $\mathrm{C} / \mathrm{Si}$ ratio of 1.0 is used in all calculations. The process pressure is $200 \mathrm{mbar}$, and $\mathrm{H}_{2}$ is used as carrier gas with a $\mathrm{Si} / \mathrm{H}_{2}$ ratio of $0.15 \%$.

\section{Results and discussion}

Growth rates are calculated twice for each simulation, since deposition of carbon and silicon are treated independently; first from the simulated deposition of carbon, assuming that there is enough silicon available to form stoichiometric $\mathrm{SiC}$; secondly from the deposition of silicon, assuming that there is enough carbon available. Comparison is made for $\mathrm{C}_{3} \mathrm{H}_{8}$ and $\mathrm{CH}_{4}$ as carbon precursors. Comparison is also made between our detailed gas-phase reaction model, and one of the more comprehensive gas-phase models previously published, by Allendorf and Kee [11]. The results are shown in Fig. 1. It is seen that using the detailed model, a large difference in carbon deposition is evident between using $\mathrm{C}_{3} \mathrm{H}_{8}$ or $\mathrm{CH}_{4}$ as precursor. The previous model, however, does not predict any large difference between the two precursors - in contrast the results are very similar.

When looking at species that contribute the most to carbon deposition, the same five species are predicted regardless of the precursor used: $\mathrm{C}_{2} \mathrm{H}_{2}, \mathrm{CH}_{3}, \mathrm{CH}_{4}, \mathrm{CH}_{2}$ and $\mathrm{C}_{2} \mathrm{H}_{4}$. Previous studies have looked at concentration profiles of different molecules in the gas-phase close to the surface, and from that conclusions about which species that are important to growth have been drawn. While it is true that the molecules contributing to growth need to be present in the gas-phase to some extent, it is not certain that the ones having the highest concentrations above the growth surface are those that in fact contribute the most to the growth, because if a molecule does contribute to the growth, it will no longer be present in the gas-phase. Therefore, a combination of concentrations and concentration gradients must be studied in order to determine this. Alternatively, as is done in the simulations here, a deposition model that completely separates the different molecules can be used.

It shall be noted that no etching effect is included in the simple surface reaction model used here. The etching effect of hydrogen does have a significant influence on the growth rate profile, as 
shown by several authors [1, 2, 3]. Etching of the deposited layer will move some material further downstream, and thereby flatten out the deposition profile somewhat. It may very well be that, even though silicon limited growth is predicted here, carbon limited growth could occur at the wafer position for $\mathrm{CH}_{4}$ as precursor, due to etching. Further work is needed to study this phenomenon.

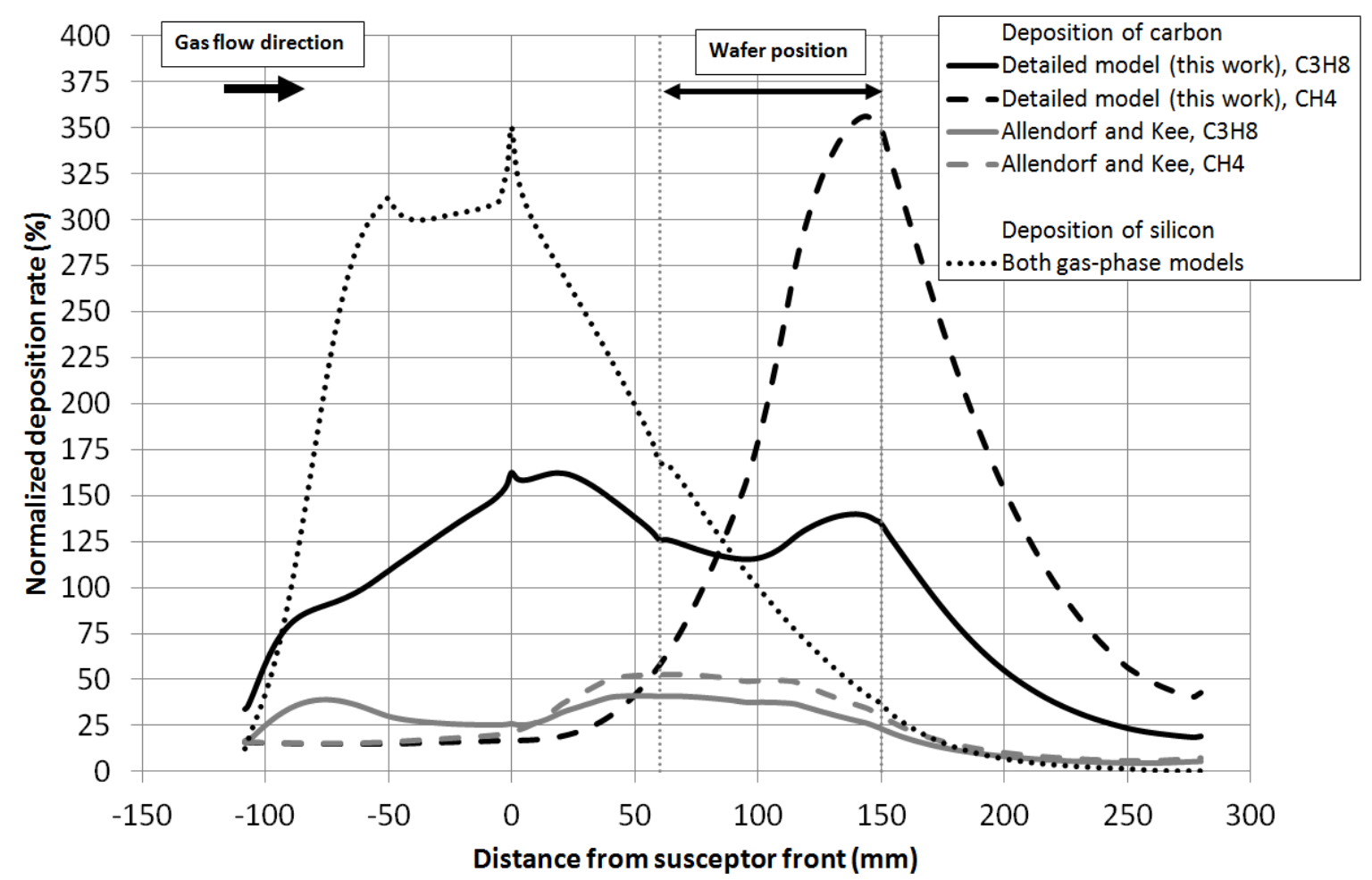

Fig. 1 Normalized predicted deposition rates of carbon and silicon for two different carbon precursors, and two different gas-phase models for hydrocarbon reactions.

Comparing with experiments. Experimentally, at the conditions used in the simulations, silicon limited growth is expected, at least for $\mathrm{C}_{3} \mathrm{H}_{8}$ as carbon precursor. In Fig. 1, silicon limited growth is predicted for the last $3 / 4$ of the wafer position area, when using the detailed model. However, the model of Allendorf and Kee predicts a carbon limited growth at the same conditions. This is in accordance with the findings in the original publication of this model [11].

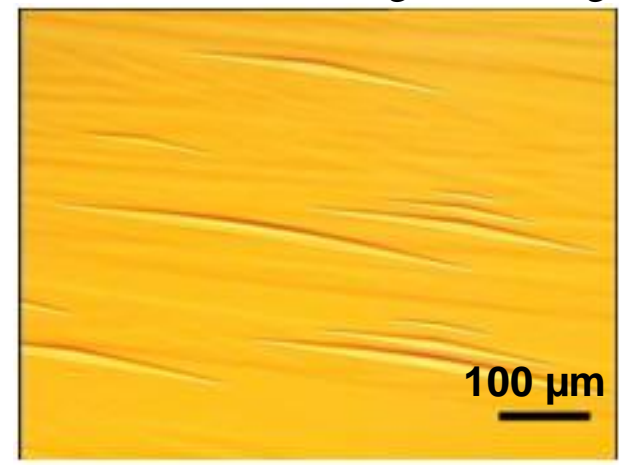

Fig. 2 Optical micrograph of epitaxial layers grown from $\mathrm{CH}_{4}$.

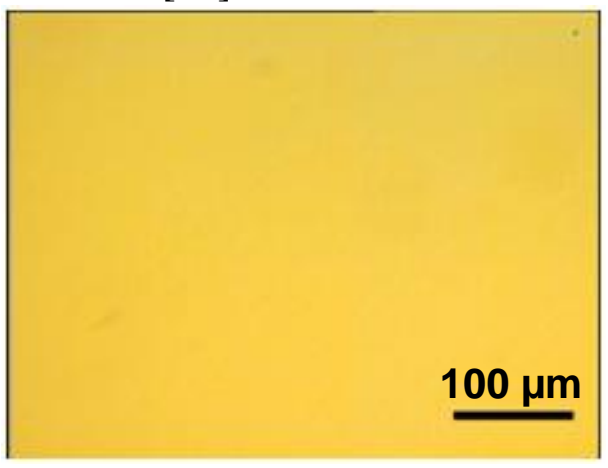

Fig. 3 Optical micrograph of epitaxial layers grown from standard precursors.

From experiments it is observed that growth rates are similar for $\mathrm{C}_{3} \mathrm{H}_{8}$ and $\mathrm{CH}_{4}$, but that the surface morphology is worse for $\mathrm{CH}_{4}$ (at the same inlet $\mathrm{C} / \mathrm{Si}$ ratio). It is also known that the morphology becomes worse at higher $\mathrm{C} / \mathrm{Si}$ ratios (for the same precursor). In Fig. 2 and Fig. 3 optical micrographs of epitaxial layers grown from different carbon precursors are shown, where the difference in surface morphology is clearly shown. One explanation, if the simulation results are correct, could be that the carbon content over the wafer area changes dramatically when $\mathrm{CH}_{4}$ is used, from relatively low rates in the upstream region to relatively high rates downstream. 
Upstream there would thus be a silicon rich environment, while there would be a carbon rich environment downstream. For $\mathrm{C}_{3} \mathrm{H}_{8}$, the carbon deposition rate is more uniform, which would imply that similar conditions prevail over the entire wafer area, making it easier to optimize process parameters for good surface morphology.

In addition, in real growth runs, more parasitic growth is observed in the wafer area when using $\mathrm{CH}_{4}$, as compared to other carbon precursors. This could also be explained by the results here; the model predicts a large amount of "extra" carbon in the wafer area that is not matched by the amount of silicon. Thus, one might expect more non-stoichiometric SiC deposition in this area.

\section{Conclusions}

It can be concluded from the results presented here that the more detailed model used in the simulations may explain some of the experimentally observed differences between the two carbon precursors studied. It is also concluded that the five most important species that act as carbon source are similar to what has been shown in the literature, except for the $\mathrm{CH}_{2}$ molecule, which has not been reported as significant earlier. Silicon limited growth is predicted by the more detailed model, whereas carbon limited growth is predicted with the previous model. The difference between the results from the two models is the predicted amount of available molecules at the growth surface.

\section{Summary}

A first step towards a general model for CVD growth of $\mathrm{SiC}$, valid for any range of process parameters and choice of precursors, is taken. Mainly the hydrocarbon chemistry has been studied by a detailed gas-phase reaction model, and comparison has been made between $\mathrm{C}_{3} \mathrm{H}_{8}$ and $\mathrm{CH}_{4}$ as carbon precursors. The results indicate that experimentally observed differences, which previous models have been unable to predict, may be explained by the new model.

\section{Acknowledgements}

Support from the Swedish Foundation for Strategic Research (project SM11-0051) is gratefully acknowledged.

\section{References}

[1] Ö. Danielsson, A. Henry, E. Janzén, J. Cryst. Growth 243 (2002) 170-184.

[2] J. Meziere, M. Ucar, E. Blanquet, M. Pons, P. Ferret, L. Di Cioccio, J. Cryst. Growth 267 (2004) 436-451.

[3] S-i. Nishizawa, M. Pons, Chem. Vap. Deposition 12 (2006) 516-522.

[4] A. Veneroni, M. Masi, Chem. Vap. Deposition 12 (2006) 562-568.

[5] W. Tsang, R. F. Hampson, J. Phys. Chem. Ref. Data, 15 (3) (1986) 1087-1279.

[6] W. Tsang, J. Phys. Chem. Ref. Data 17 (2) (1988) 887-951.

[7] A. Veneroni, F. Omarini, M. Masi, S. Leone, M. Mauceri, G. Pistone, G. Abbondanza, Cryst. Res. Technol. 40 (11) (2005) 972-975.

[8] C. Hallin, I. G. Ivanov, T. Egilsson, A. Henry, O. Kordina, E. Janzén, J. Cryst. Growth 183 (1998) 163-174.

[9] S. Leone, H. Pedersen, A. Henry, O. Kordina, E. Janzén, J. Cryst. Growth 312 (2009) 24-32.

[10] P. Ho, M. E. Coltrin, W.G. Breiland, J. Phys. Chem. 98 (1994) 10138-10147.

[11] M. D. Allendorf, R. J. Kee, J. Electrochem. Soc. 138 (3) (1991) 841-852. 